between rs727139 (KCNH8) on chromosome 3 and rs11167496 (PDGFRB) on chromosome $5\left(\mathrm{p}=2.45 \times 10^{-8}\right)$. Analysis of subsets of SNPs pre-selected based on their nominal association with CAD $(p<0.05)$ or molecular functionality (non-synonymous SNPs) did not contribute more significant findings than investigation of random set of SNPs.

Conclusion Our analysis suggests that common SNP-SNP interactions are unlikely to account for a large proportion of the missing heritability of $\mathrm{CAD}$.

\section{CLINICAL AND FINANCIAL REPERCUSSIONS OF THE MARCH 2010 NATIONAL INSTITUTE FOR HEALTH AND CLINICAL EXCELLENCE (NICE) GUIDELINE "CHEST PAIN OF RECENT ONSET" ON THE RAPID ACCESS CHEST PAIN CLINIC (RACPC)}

doi:10.1136/heartjnl-2011-300198.56

T Rogers, S Claridge, K Al Fakih. University Hospital Lewisham, London, UK

Background The RACPC is a well-established "one-stop" service, with goal to identify patients with stable chest pain due to coronary artery disease (CAD) and quickly reassure those with non-cardiac pain. In March 2010, NICE published a new guideline, which advocates assessing likelihood of $\mathrm{CAD}$ based on age, gender, history and risk factors (RF). If estimated likelihood is $>60 \%$, invasive coronary angiography (ICA) is recommended as the first-line diagnostic investigation. If estimated likelihood is $30 \%-60 \%$, functional imaging is recommended. If estimated likelihood is $<30 \%$, CT calcium scoring CT coronary angiography (CTCA) is recommended. Significantly, the guideline discourages the use of ETT to diagnose or exclude stable angina in patients without known CAD.

Methods 167 consecutive patients referred to RACPC between October 2009 and March 2010 were retrospectively assessed for likelihood of CAD according to the new NICE guideline. Choice of investigations and eventual outcome (confirmed CAD vs no evidence of $\mathrm{CAD}$ ) were compared between subgroups defined by estimated likelihood of CAD. An economic analysis of cost of investigation per patient was undertaken using current Payment by Results national tariffs.

Results Our patient population had a high prevalence of RF with $38.1 \%$ having a total of three or more RF. Consequently $23.2 \%$ of patients had an estimated likelihood of CAD of $<30 \% .27 .4 \%$ had an estimated likelihood of $30 \%-60 \% .49 .4 \%$ had an estimated likelihood of $>60 \%$. $7.2 \%$ of patients were lost to follow-up. $14.4 \%$ of patients were ultimately confirmed to have CAD on ICA, which correlated with pre-test estimated likelihood. $6 \%$ of patients with likelihood $<30 \%$, $8.7 \%$ of those with likelihood $30 \%-60 \%$ and $23.2 \%$ of those with likelihood $>60 \%$ were confirmed to have CAD. Average cost of investigation per patient was $£ 528$. A negative ETT resulted in average cost per patient of $£ 347$. An inconclusive ETT resulted in higher cost (£728) as did inability to exercise (£435) due to the need for further investigations. A positive ETT resulted in average cost of $£ 1174$ due to the high cost of ICA. Were the NICE guideline strictly applied to our patient population, average cost per patient would have been substantially higher at $£ 838$ ( $£ 362$ per patient if likelihood $<30 \%$, £566 if likelihood $30 \%-60 \%$ and $£ 1218$ if likelihood $>60 \%$ ). Overall this corresponds to a $60 \%$ increase in cost. Conclusion The 2010 NICE guideline appears to significantly overestimate the true risk in our patient population. Were the guideline strictly applied, almost half of our patients would proceed to ICA as a first-line investigation, but many of them would be found to have unobstructed coronary arteries. As ICA is an expensive investigation, this would inevitably result in a significant increase in average cost per patient. Relatively few patients would be eligible for CTCA, which is an excellent non-invasive "rule-out" test for $\mathrm{CAD}$ and relatively inexpensive compared with other investigations.

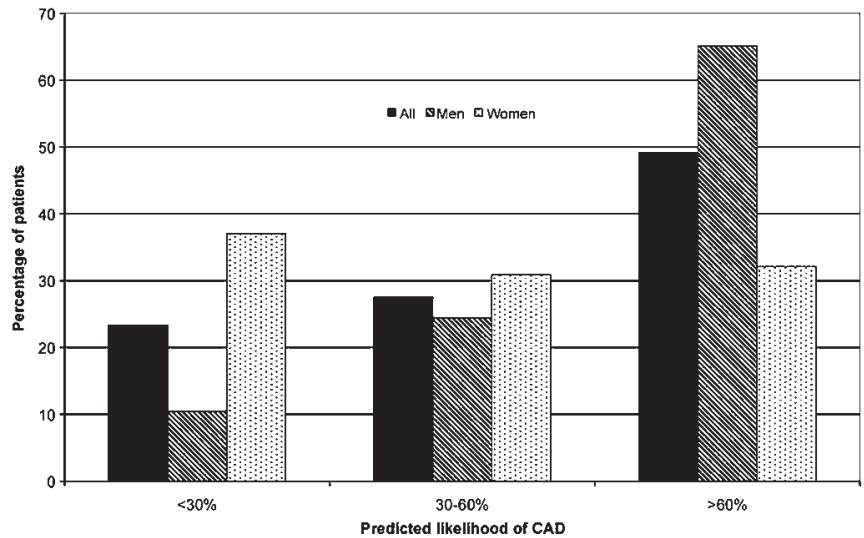

Abstract 56 Figure 1

Abstract 56 Table 1

\begin{tabular}{llll}
\hline & All & Men & Women \\
\hline Numbers (\%) & 167 & $86(51.5)$ & $51(48.5)$ \\
Age (mean \pm SD) & $56.0 \pm 12.6$ & $55.2 \pm 13.3$ & $57.0 \pm 11.8$ \\
Diabetes (\%) & $29(17.4)$ & $15(17.4)$ & $14(17.3)$ \\
Hypertension (\%) & $72(43.1)$ & $40(46.5)$ & $32(39.5)$ \\
Hypercholesterolaemia (\%) & $100(59.9)$ & $53(61.6)$ & $47(58.0)$ \\
Family history (\%) & $58(34.7)$ & $28(32.6)$ & $30(37.0)$ \\
Smoking (\%) & $87(52.1)$ & $54(62.8)$ & $33(40.7)$ \\
Systolic BP (mean \pm SD) / & $134 \pm 21 /$ & $131 \pm 18 /$ & $137 \pm 23 /$ \\
Diastolic BP (mean \pm SD) & $79 \pm 11$ & $80 \pm 11$ & $78 \pm 10$ \\
Fasting glucose (mean \pm SD) & $5.2 \pm 1.0$ & $5.7 \pm 1.0$ & $5.3 \pm 1.1$ \\
Total cholesterol (mean \pm SD) & $5.17 \pm 1.04$ & $5.06 \pm 1.06$ & $5.29 \pm 1.01$ \\
LDL (mean \pm SD) / HDL & $3.19 \pm 0.90 /$ & $3.14 \pm 0.90 /$ & $3.33 \pm 0.86 /$ \\
(mean \pm SD) & $1.30 \pm 0.43$ & $1.23 \pm 0.46$ & $1.38 \pm 0.38$ \\
BMI (mean \pm SD) & $30.1 \pm 6.0$ & $29.4 \pm 4.9$ & $30.9 \pm 6.9$ \\
\hline
\end{tabular}

Abstract 56 Table 2

\begin{tabular}{lll}
\hline & $\begin{array}{l}\text { Average cost per } \\
\text { patient prior to NICE } \\
\text { guideline implementation }\end{array}$ & $\begin{array}{l}\text { Average cost per } \\
\text { patient were NICE } \\
\text { guideline strictly } \\
\text { implemented }\end{array}$ \\
\hline Predicted likelihood $<30 \%$ & $£ 324$ & $£ 362$ \\
Predicted likelihood $30 \%-60 \%$ & $£ 467$ & $£ 566$ \\
Predicted likelihood $>60 \%$ & $£ 661$ & $£ 1218$ \\
OVERALL AVERAGE COST PER & $£ 528$ & $£ 838$ \\
PATIENT & & \\
\hline
\end{tabular}

57 THE IMPACT OF PREOPERATIVE RENAL DYSFUNCTION AND THERAPY TYPE IN PATIENTS WITH TYPE 2 DIABETES UNDERGOING CORONARY ARTERY BYPASS SURGERY

doi:10.1136/heartjnl-2011-300198.57

A Menon, J Hodson, D Pagano, J Mascaro, I C Wilson, S J Rooney, T R Graham, R S Bonser. University Hospital Birmingham, Birmingham, UK

Introduction There is limited data addressing the impact of preoperative renal dysfunction in type 2 diabetics (T2DM) undergoing firsttime coronary artery bypass surgery (CABG); specifically exploring the influence of diabetic management (oral hypoglycaemic $(\mathrm{OH})$ and insulin therapy (IN)). We assessed the impact of preoperative renal status and diabetic management on the post operative renal status, morbidity, 30-day and long-term survival in T2DM-CABG.

Methods We reviewed prospectively accrued data from 1/1/1999 to $31 / 12 / 2009$. Pre and 4 to 5 -day postoperative creatinine clearance 
(CrCl) was calculated using Cockcroft-Gault formula. Patients were subgrouped into 5 grades based on preoperative $\mathrm{CrCl}$; Group I $\mathrm{CrCl} \geq 90 \mathrm{ml} / \mathrm{min}$; II $60-89$; III $30-59 ;$ IV $15-29 ; \mathrm{V}<15$ or haemodialysis. Late Kaplan-Meier survival data (compared by log rank method), censored at 1/10/2009 were obtained from the UK $\mathrm{CCAD}$. Surgical morbidity outcomes included re-exploration for bleeding, stroke (type 1 deficit) and low cardiac output state (LCOS) requiring inotropes \pm intra-aortic balloon counterpulsation were compared using Fisher's Exact tests.

Results 1215 patients (921 males) with a mean age of 64 years (31-89 years) underwent CABG; 742 on $\mathrm{OH}$ and 472 on IN. Preoperative renal status in the groups were Group I -209(17\%), II-584 (48\%), III-387(32\%), IV-26(2\%) and V (8(1\%). Similar percentages in each group had $\geq 1$ grade deterioration of renal function postoperatively $19 \%, 18 \%, 16 \%$ and $23 \%$ (grades I-IV respectively; $\mathrm{p}=0.470$ ). When examined as a continuous variable, higher preoperative $\mathrm{CrCl}$ correlated with a better postoperative improvement in $\mathrm{CrCl}(\mathrm{r}=0.073, \mathrm{p}=0.012$ Spearman Rank). Overall 30-day mortality was $3.33 \%$ (CI 2.32 to $4.34 \%$ ) and was not different by group I-3.37\% (CI 0.92 to 5.82 ), II $-2.09 \%$ (CI 0.92 to $3.26 \%$ ), III $4.92 \%$ (CI 2.76 to $7.08 \%$ ), IV $8 \%$ (CI 0 to $18.6 \%$ ) and Stage V $0 \%$ (CI 0 to $0.4 \%$; $\mathrm{p}=0.101$ ) or by therapy type; $(p=0.411)$. IN patients had similar preoperative renal function (median $\mathrm{CrCl} 66.8$ vs 68.6; $\mathrm{p}=0.828$ ) but a higher rate of postoperative renal deterioration ( 53.3 vs $46.7 \%, p<0.001$ ). Stroke $(p=1.000)$, bleeding $(p=0.755)$ and LCOS $(p=0.335)$ incidence were not different between therapy type. Overall mean survival was 9 years (CI 8.7 to 9.2 years) and was not different by renal function grade $(p=0.612)$. However, IN patients had shorter mean survival 8.7 (8.3 to 9.0) vs OH 9.1(8.8 to 9.4) years; $p=0.03$.

Conclusions In T2DM-CABG, $36 \%$ of patients have $\mathrm{CrCl}<60 \mathrm{ml} / \mathrm{min}$. Higher $\mathrm{CrCl}$ protects against postoperative renal deterioration. Renal dysfunction does not appear to affect hospital outcome or survival. However, preoperative IN requirement increases the risk of renal dysfunction and is associated with worse longer-term survival.

\section{TEMPORAL EVALUATION OF REFERRAL FOR AND LONG- TERM SURVIVAL FROM CARDIAC REHABILITATION FOR ACUTE MYOCARDIAL INFARCTION}

\section{doi:10.1136/heartjnl-2011-300198.58}

${ }^{1} \mathrm{C}$ L Lewinter, ${ }^{1} \mathrm{M}$ B Bland, ${ }^{2} \mathrm{P}$ D Doherty, ${ }^{1} \mathrm{~B}$ L Lewin, ${ }^{3} \mathrm{~A}$ S H Hall, ${ }^{4} \mathrm{C}$ P G Gale. ${ }^{1}$ University of York, York, UK; ${ }^{2}$ York St John University, York, UK; ${ }^{3}$ Yorkshire Heart Centre, Leeds, UK; ${ }^{4}$ University of Leeds, Leeds, UK

Background Cardiac rehabilitation (CR) is a cost-effective, evidencebased approach to managing heart disease. Rates of uptake have and continue to vary despite recommendations from the NSF for CHD and NICE. The Evaluation of the Management and Methods of Acute Coronary Events (EMMACE) 1 and 2 studies are 2 large prospective multi-centre registries of care of acute coronary syndromes (ACS) in Yorkshire undertaken in 1995 and 2003 in respectively. We studied the temporal changes in referral for and long-term survival from $\mathrm{CR}$ in patients who were admitted to hospital with an acute myocardial infarction (AMI).

Methods Baseline characteristics were described as numbers (\%) or as means with IORs. For Continuous variables, the Kruskal Wallis test was used for comparisons. Discrete variables were assessed by the $\chi^{2}$ test. Unadjusted relative risk ratios (RRR) were calculated to assess mortality after referral for CR. Kaplan-Meier (KM) curves compared unadjusted survival stratified by CR referral and EMMACE study. Log rank tests compared the survival estimates. Sex, age, STEMI, heart failure, diabetes, COPD and mini-GRACE score, revascularisation, reperfusion, ACE-inhibitors, $\alpha$-blockers, statins, anti-platelet agents and admitting cardiologist were regressed (backward logistic, $p<0.10$ and goodness of fit with a group of 10) on CR referral and represented as $95 \%$ CI OR. A Cox proportional model (Model 1: mini-GRACE score, Model 2: sex, age, STEMI, heart failure, diabetes, COPD,
EMMACE risk score, revascularisation, reperfusion, ACE-inhibitor, $\alpha$ blocker, statins, anti-platelet agent, admitting cardiologist) was used to compare the temporal long-term survival estimates (all cause mortality) by CR referral.

Results 4341 had AMI. CR referral was 44\% in 1995 and 59\% in 2003 $(\mathrm{p}<0.001)$. CR referral was associated with reduced mortality in 2003 (RRR, 95\%CI: 0.54; 0.50 to 0.60), but was not in 1995 (1.02; 0.96 to 1.09). Unadjusted survival for patients not referral for CR in 1995 was similar to that for patients referred for CR in 1995; (Abstract 58 figure.1). For those referred for $\mathrm{CR}$, the mean mini-GRACE score for CR referrals was lower in 2003 than 1995; 0.53 and 0.72, $\mathrm{p}<0.001$ After adjustment using the min-GRACE score (Model 1), the impact (HR, 95\% CI) of CR referral was $0.63,0.55$ to 0.73 in 2003 and 1.07 , 0.92 to 1.3 in 1995. After adjustment using Model 2, the impact (HR, 95\% CI) of CR referral was $0.57,0.48$ to 0.66 in 2003 and 1.31 , 1.04 to 1.60 in 1995

Conclusion Between 1995 and 2003, referral for CR increased and became a significantly important factor contributing to reduced mortality rates post-AMI. This is despite the differences in patient and treatment factors between the 2 studies periods. Even so, rate of referral for CR remain sub-optimal.

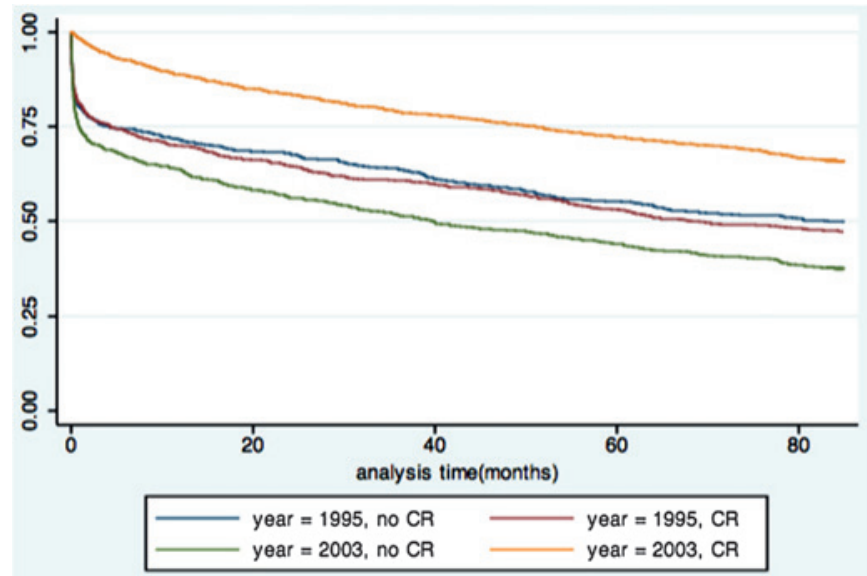

Abstract 58 Figure 1 Kaplan-Meier survival estimates.

\section{SHORT TERM ELEVATION OF CHOLESTEROL LEVEL IN NEONATAL LIFE AND LONG TERM CHANGES IN AORTIC STIFFNESS: INSIGHTS FROM USE OF INTRAVENOUS LIPIDS}

doi:10.1136/heartinl-2011-300198.59

${ }^{1} \mathrm{~A} J$ Lewandowski, ${ }^{1} \mathrm{M}$ Lazdam, ${ }^{1} \mathrm{E}$ Davis, ${ }^{1} \mathrm{R}$ Poole, ${ }^{1} \mathrm{~J}$ Diesch, ${ }^{1} \mathrm{~J}$ Francis, ${ }^{1} \mathrm{~S}$ Neubauer, ${ }^{2} \mathrm{~A}$ Lucas, ${ }^{2} \mathrm{~A}$ Singhal, ${ }^{1} \mathrm{~B}$ Kelly, ${ }^{1} \mathrm{P}$ Leeson. ${ }^{1}$ Cardiovascular Medicine, University of Oxford, Oxford, UK; ${ }^{2}$ Institute of Child Health, University College London, London, UK

Introduction Offspring born to hypercholesterolaemic mothers have increased fatty streak formation in the fetal aorta, which persists into adolescence. To understand whether exposure to elevated cholesterol in early life, independent of a maternal history of hypercholesterolaemia, also has a long-term impact on the cardiovascular system we studied the vascular phenotype of adults in whom cholesterol levels were artificially elevated for a short period postnatally.

Methods We prospectively followed-up 102 subjects born premature now aged 23 to 28 years. Individuals exposed to maternal hypercholesterolaemia were excluded. 18 received intravenous (IV) lipids during the first nine weeks of life and were matched 2:1 for pregnancy and early life complications, age, sex, birthweight and gestational age with controls that did not receive IV lipids. Aortic pulse wave velocity (aPWV), regional aortic distensibility, left ventricular mass and ejection fraction were determined by cardiovascular 